\title{
Colagenomas: manifestación cutánea de neoplasia endocrina múltiple tipo 1 (MEN 1)
}

\author{
Laura Pulgarín'; Simón Gallo²; Natalia Giraldo³; Carlos Montealegre; Alejandro \\ Roman-González ${ }^{5}$
}

\section{RESUMEN}

La neoplasia endocrina múltiple tipo 1 (MEN 1) es un síndrome hereditario raro caracterizado por la aparición de tumores de paratiroides, páncreas e hipófisis anterior, que en algunos casos se acompaña de neoplasias cutáneas benignas, como angiofibromas y colagenomas, cuya aparición puede preceder a las manifestaciones endocrinológicas.

Se presenta el caso de una paciente de 23 años, en quien se diagnosticó hiperparatiroidismo primario, enfermedad de Cushing, tumor neuroendocrino de páncreas y múltiples colagenomas, que aparecieron antes de las manifestaciones hormonales.

PALABRAS CLAVE: Enfermedades de la piel y tejido conjuntivo; Neoplasias cutáneas; Neoplasia endocrina múltiple tipo 1.

1. Médico general, Universidad de Antioquia. Residente de Dermatología, Universidad del Valle. ORCID https://orcid.org/oooo-00o1-9609-9807

2. Médico general. Residente de Dermatología, Centro de Investigaciones Dermatológicas CIDERM, Sección de Dermatología, Facultad de Medicina, Universidad de Antioquia. ORCID https://orcid.org/oooo-0003-2473-2516

3. Médico general. Residente de Dermatología, Centro de Investigaciones Dermatológicas CIDERM, Sección de Dermatología, Facultad de Medicina, Universidad de Antioquia. ORCID https://orcid.org/oooo-0003-0279-8709

4. Médico dermatólogo, Centro de Investigaciones Dermatológicas CIDERM, Sección de Dermatología, Facultad de Medicina, Universidad de Antioquia. ORCID https://orcid.org/oooo-0001-8496-3717

5. Médico endocrinólogo, Universidad de Antioquia, Hospital Universitario San Vicente Fundación. ORCID https://orcid.org/oooo-0001-5942-1035

Correspondencia: Laura Manuela Pulgarín Zapata; email: lauramanuelapulgarin@gmail.com

Recibido: $13 / 02 / 20$; aceptado: $02 / 05 / 20$

Cómo citar: Pulgarín L; Gallo S; Giraldo N; Montealegre C; Roman-González A. Colagenomas: manifestación cutánea de neoplasia endocrina múltiple tipo 1 (MEN 1). Rev Asoc Colomb Dermatol. Vol 28(3): julio - septiembre, 2020, 269-273.

DOI: https://doi.org/10.29176/2590843X.1539

Financiación: ninguna, conflictos de interés: ninguno 


\section{COLLAGENOMAS: CUTANEOUS MANIFESTATION OF MULTIPLE ENDOCRINE NEOPLASIA TYPE 1 (MEN 1)}

\section{SUMMARY}

Multiple endocrine neoplasia type 1 is a rare hereditary syndrome characterized by parathyroid, anterior pituitary and pancreatic tumors, that may also be associated with benign cutaneous neoplasms like angiofibromas and collagenomas, that can precede endocrine manifestations.

We present the case of a 23-year-old female patient with primary hyperparathyroidism, Cushing disease and a pancreatic neuroendocrine tumor associated with multiple collagenomas that appeared before systemic symptoms.

KEY WORDS: Skin and connective tissue diseases; Skin neoplasms; Multiple endocrine neoplasia type 1.

\section{INTRODUCCIÓN}

La neoplasia endocrina múltiple tipo 1 (MEN 1), también conocida como síndrome de Wermer o adenomatosis endocrina múltiple, es una enfermedad con transmisión autosómica dominante dada por las alteraciones en el gen MEN1, ubicado en el cromosoma 11q13, que codifica la menina, una proteína supresora de tumores. Es una patología de baja frecuencia, que se presenta hasta en 1 por cada 30.000 personas, sin predilección de raza o sexo y suele manifestarse entre la cuarta y quinta década de la vida.

El síndrome se caracteriza por presentar hiperparatiroidismo primario en el $95 \%$ de los casos, tumores neuroendocrinos gastroenteropancréaticos en el 30\% al 80\% y adenomas de la hipófisis anterior en el 15\% al 90\% de los casos. Puede presentarse de forma esporádica, definida como la presencia de 2 de las 3 neoplasias mencionadas, o de forma familiar: paciente con diagnóstico de MEN 1 y un familiar de primer grado con al menos una neoplasia característica del síndrome ${ }^{(1)}$. A pesar de que históricamente se le ha conocido como una patología de neoplasias endocrinas, desde 1997 se sabe que presenta también neoplasias cutáneas benignas: se han reportado angiofibromas hasta en un $88 \%$, colagenomas en un $72 \%$ y lipomas en un $34 \%$ de los pacientes ${ }^{(2,3)}$, en los cuales se ha demostrado deleción alélica en la piel afectada ${ }^{(4)}$. Por otro lado, pareciera ser que el gen MEN1 desempeña un papel en la génesis tumoral de un pequeño subgrupo de melanoma, lo que podría estar relacionado con mayor prevalencia de este tumor maligno en este grupo de pacientes ${ }^{5}$. Se ha encontrado que las manifestaciones cutáneas, en especial los angiofibromas y colagenomas, pueden aparecer de forma temprana, precediendo las manifestaciones endocrinológicas de la enfermedad ${ }^{(2)}$. Asgharian y colaboradores encontraron que al combinar más de tres angiofibromas y un colagenoma se tiene una sensibilidad del $75 \%$ y una especificidad del $95 \%$ para el diagnóstico de la enfermedad en sujetos con MEN1 y gastrinoma; este criterio tiene mayor sensibilidad que la enfermedad hipofisaria o suprarrenal y es comparable al hiperparatiroidismo en algunos estudios en el mismo tipo de pacientes ${ }^{(3)}$.

\section{CASO CLÍNICO}

Se presenta el caso de una mujer de 23 años, costurera, soltera y sin hijos, con unos antecedentes de síndrome de ovario poliquístico y obesidad desde los 13 años. Consultó por un dolor abdominal intenso y múltiples episodios de vómito. En la revisión por sistemas refirió tener múltiples lesiones cutáneas en el abdomen y los pliegues, de aparición progresiva, que comenzaron cuatro años atrás, asociadas a redistribución grasa, fatiga, constipación y un episodio de nefrolitiasis, por lo que le realizaron una ecografía abdominal y una tomografía axial computarizada, donde se evidenciaron una masa suprarrenal izquierda y otra pancreática. Durante dicha hospitalización se le formuló el diagnóstico de enfermedad de Cushing, hiperparatiroidismo primario y un tumor neuroendocrino del páncreas.

Al examen físico se le encontró un índice de masa corporal de $46 \mathrm{~kg} / \mathrm{m}^{2}$, una giba de búfalo y una obesidad de distribución centrípeta con numerosas estrías 
gruesas de color piel. Además, unas placas hiperpigmentadas de color marrón, con apariencia aterciopelada y acentuación del reticulado cutáneo en el cuello y las axilas, compatibles con una acantosis nigricans. Se observaron también más de cien pápulas y nódulos sésiles, de color piel, y consistencia firme, principalmente en el cuello, las axilas y el abdomen, concordantes con colagenomas (figura 1), por lo que se tomó una biopsia de piel que confirmó la impresión diagnóstica (figuras 2 y 3 ).

Ante la presencia de enfermedad de Cushing, hiperparatiroidismo primario, tumor neuroendocrino de pán- creas y múltiples colagenomas, se hace el diagnóstico de un MEN 1. El estudio genético ya fue ordenado y se encuentra pendiente el resultado. La paciente niega tener antecedentes familiares de la enfermedad.

\section{DISCUSIÓN}

La neoplasia endocrina múltiple tipo 1 (MEN 1) es una enfermedad poco frecuente, caracterizada por hiperplasia paratiroidea, tumores pancreáticos endocrinos, adenomas de la hipófisis anterior ${ }^{(1)}$ y manifestaciones cutáneas, entre las que se incluyen: angiofibromas, co-
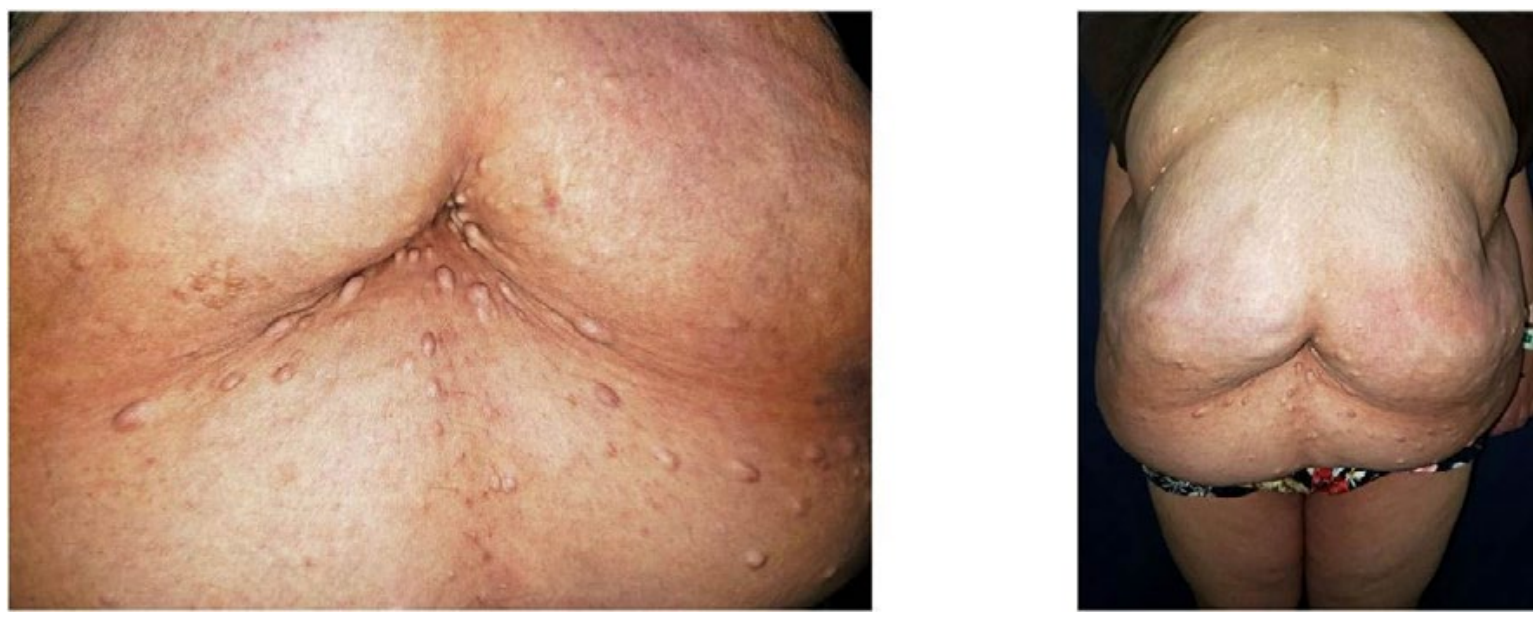

Figura 1. A) Pápulas y nódulos sésiles, eucrómicos, de consistencia firme y de predominio periumbilical. B) Abundante panículo adiposo, obesidad de predominio central, asociado a estrías blancas delgadas y a pápulas y nódulos sésiles.

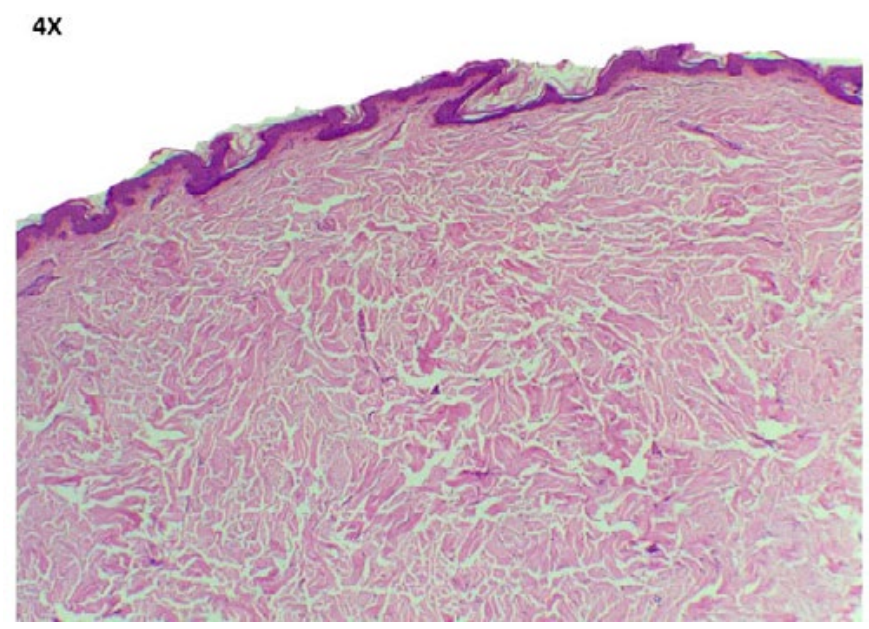

Figura 2. Coloración de hematoxilina-eosina con aumento $4 \mathrm{X}$. Estrato córneo delgado, epidermis con aplanamiento de la red de crestas. Dermis con engrosamiento y formación de lesión nodular bien delimitada, caracterizada por proliferación de fibras de colágeno gruesas organizadas al azar, sin atipia. Sin hallazgos sugestivos de malignidad. 


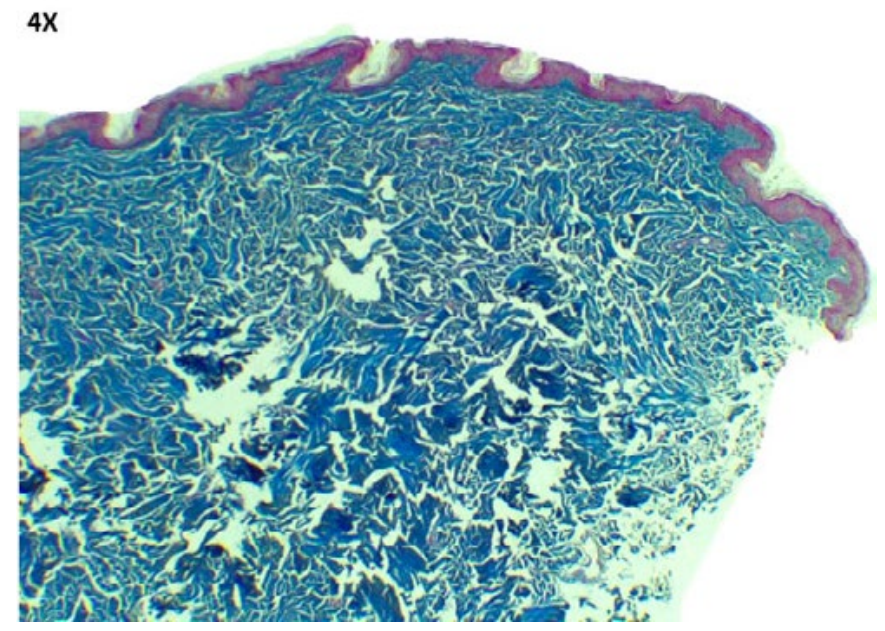

Figura 3. Tricrómico de Masson con aumento 4X. Fuertemente positivo para fibras de colágeno.

lagenomas, lipomas, melanomas y manchas café con leche ${ }^{(2,3)}$. Esta tiene un patrón de herencia autosómica dominante con alta penetrancia, pero cuyo genotipo no se correlaciona con el fenotipo; así pues, dos pacientes con la misma alteración genética podrían mostrar cuadros clínicos completamente distintos ${ }^{(6)}$.

Debido a su baja prevalencia, a su amplia variabilidad clínica, a que el estudio genético no siempre está disponible y al alto costo de este, se ha propuesto que las alteraciones cutáneas podrían servir como un indicador clínico de la enfermedad antes de la aparición de las neoplasias. Darling y colaboradores ${ }^{(2)}$ propusieron desde 1997 la presencia de angiofibromas, colagenomas y lipomas como lesiones sugestivas de MEN 1, que podian presentarse incluso en períodos presintomáticos. Asgharian y colaboradores (3) analizaron dichas neoplasias benignas cutáneas en 103 pacientes con diagnóstico de gastrinomas, comparando la frecuencia de presentación en aquellos con MEN 1 y los que presentaban gastrinomas esporádicos; la presentación de colagenomas fue del $62 \%$ contra el 5\%, respectivamente. Dicho estudio concluyó, además, que los pacientes que presentaban colagenomas asociados a MEN 1 presentaban múltiples lesiones y que en el $83 \%$ presentaban al menos 4 nódulos. Dichos autores proponen que la presentación de colagenomas en un paciente con síndrome de Zollinger-Ellison podría tener una sensibilidad del $63 \%$ y una especificidad del $95 \%$ para MEN 1, y que dicha especificidad aumenta al $97 \%$ cuando se presentan más de 3 lesiones.

Vidal y colaboradores (7) analizaron la frecuencia de lesiones cutáneas en los pacientes con diagnóstico de
MEN 1, contra familiares en primer grado de dichos pacientes, no portadores de la alteración genética. Los autores encontraron que los pacientes con MEN 1 presentan alteraciones cutáneas en el 55\% contra el 25\% de sus familiares. Las lesiones que más encontraron fueron: lipomas en el 33\% y angiofibromas en el $22 \%$ de los casos, y no reportaron pacientes con colagenomas. Es de tener presente que la muestra del estudio fue pequeña (9 pacientes con MEN 1) y esto podría explicar la diferencia con lo encontrado en otras series.

Es importante también tener en cuenta que los factores étnicos podrían desempeñar un papel en la presentación de las manifestaciones cutáneas asociadas a MEN 1, como lo reportado por Sakurai y colaboradores ${ }^{(6)}$, quienes encontraron angiofibromas en el $43 \%$ de 28 pacientes con diagnóstico de MEN 1, lo cual es mucho menor que el 88\% reportado por Darling y colaboradores en una cohorte caucásica de 32 pacientes ${ }^{(2)}$. Además, reportaron que los pacientes japoneses presentaron un menor número de lesiones con respecto a la cohorte caucásica.

Por lo anteriormente mencionado, es importante que el médico general, el endocrinólogo y el dermatólogo tengan presente que dichas lesiones cutáneas podrían ser la primera manifestación de una enfermedad de alta morbimortalidad como el MEN 1. Existe incluso un caso reportado por Roman y colaboradores ${ }^{(8)}$, donde el paciente consultó por alteraciones cutáneas y se hizo el diagnóstico genético antes de la aparición de las neoplasias. Realizar un diagnóstico temprano permite al paciente mejorar su calidad de vida, su expectativa de vida y hacerle consultoría genética a él y su familia. 


\section{Puntos clave}

- La neoplasia endocrina múltiple tipo 1 (MEN 1) se acompaña de neoplasias cutáneas benignas, como angiofibromas y colagenomas, cuya aparición puede preceder a las manifestaciones endocrinológicas.

- Los hallazgos dermatológicos consistentes en más de tres angiofibromas y un colagenoma tienen una sensibilidad del $75 \%$ y una especificidad del 95\% para el diagnóstico de MEN 1 asociada a gastrinoma.

- La búsqueda de las anomalías cutáneas descritas surge como una opción económica y de fácil implementación, que permitiría el diagnóstico precoz de la enfermedad y la asesoría genética temprana.

\section{CONCLUSIÓN}

El MEN 1 es un síndrome clínico de baja prevalencia y alta heterogeneidad clínica, que puede manifestarse, inicialmente, mediante neoplasias cutáneas benignas. Presentamos el caso de una paciente con lesiones cutáneas asintomáticas, compatibles con colagenomas, que aparecieron al menos 2 años antes que los primeros síntomas atribuibles a sus múltiples neoplasias. Fue valorada por varios médicos en dicho intervalo de tiempo y no se sospechó la posible etiología genética, probablemente debido a la baja prevalencia de la enfermedad y al desconocimiento por parte del personal médico de la correlación entre los hallazgos cutáneos y el MEN 1. Debido a los altos costos y a la poca disponibilidad de pruebas diagnósticas tempranas de MEN 1, la búsqueda de anomalías cutáneas surge como una opción económica y de fácil implementación, que permitiría diagnósticos precoces y asesoría genética temprana.

\section{REFERENCIAS}

1. Marini F, Falchetti A, Del Monte F, Carbonell Sala S, Gozzini A, Luzi E, et al. Multiple endocrine neoplasia type 1. Orphanet J Rare Dis. 2006;1:38. doi: 10.1186/1750-1172-1-38

2. Darling TN, Skarulis MC, Steinberg SM, Marx SJ, Spiegel AM, Turner M. Multiple Facial Angiofibromas and Collagenomas in Patients With Multiple Endocrine Neoplasia Type 1. Arch Dermatol. 1997;133(7):853-7. doi:10.1001/archderm.1997.03890430067009
3. Asgharian B, Turner ML, Gibril F, Entsuah L, Serrano J, Jensen R. Cutaneous Tumors in Patients with Multiple Endocrine Neoplasm Type 1 (MEN1) and Gastrinomas: Prospective Study of Frequency and Development of Criteria with High Sensitivity and Specificity for MEN1. J Clin Endocrinol Metab. 2004;89 (11):5328-36. doi: 10.1210/jc.2004-0218

4. Pack S, Turner ML, Zhuang Z, Vortmeyer AO, Boni R, Skarulis M, et al. Cutaneous tumors in patients with multiple endocrine neoplasia type 1 show allelic deletion of the MEN1 gene. J Invest Dermatol. 1998;110(4):438-40. doi: 10.1046/j.15231747.1998.00140.x

5. Nord B, Platz A, Smoczynski K, Kytölä S, Robertson $\mathrm{G}$, Calender A, et al. Malignant melanoma in patients with multiple endocrine neoplasia type 1 and involvement of the MEN1 gene in sporadic melanoma. Int J Cancer. 2000;87(4):463-7. doi: 10.1002/1097-0215(20000815)87:4<463::AIDIJC1>3.0.CO;2-8

6. Sakurai A, Matsumoto K, Ikeo Y, Nishio SI, Kakizawa T, Arakura F, et al. Frequency of facial angiofibromas in Japanese patients with multiple endocrine neoplasia type 1. Endocr J. 2000;47(5):569-73. doi: 10.1507/endocrj.47.569

7. Vidal A, Iglesias MJ, Fernández B, Fonseca E, Cordido F. Cutaneous lesions associated to multiple endocrine neoplasia syndrome type 1. J Eur Acad Dermatol Venereol. 2008;22(7):835-8. doi: 10.1111/j.1468-3083.2008.02578.x

8. Roman JW, Logemann NF, Adams E. Incidental angiofibromas prompt a diagnosis of multiple endocrine neoplasia type-1 (MEN-1). Dermatol Online J. 2014;20(9):13030/qt4kq2z97b 